2 Naiman A. Khan, PhD, RD ${ }^{1}$, Lauren B. Raine, BS ${ }^{1}$, Eric S. Drollette, BS ${ }^{1}$, Mark R. Scudder, $3 \mathrm{BS}^{1}$, Charles H. Hillman, $\mathrm{PhD}^{1}$

4

5 6

${ }^{1}$ Department of Kinesiology and Community Health, University of Illinois, Urbana, IL

Author last names: Khan, Raine, Drollette, Scudder, Hillman

Keywords: diet, cognitive flexibility, children, obesity, aerobic fitness

Corresponding author: Naiman A. Khan, $\mathrm{PhD}$, RD, Neurocognitive Kinesiology Laboratory, Department of Kinesiology \& Community Health, University of Illinois, 313 Louise Freer Hall, MC-052, 906 S. Goodwin Avenue, Urbana, IL 61801. Telephone: 217-333-3893. Fax: 217-2447322. Email: nakhan2@illinois.edu

Funding Source: This study was supported by NIH grants (HD055352) and USDA NIFA grant (2011-67001-30101). 
Childhood represents a period marked by extensive changes in both brain structure and function $(1,2)$. Therefore, identification of health factors - lifestyle behaviors and markers of

27 physiological health - associated with childhood cognitive function has important implications

28 for not simply children's cognitive health, but cognitive function throughout the lifespan. This is 29 particularly important given the current obesogenic environment, characterized by reduced opportunities for physical activity and concomitant consumption of calorically dense but nutritionally poor diets. Emerging literature suggests that specific aspects of childhood cognitive function are selectively related to health factors such as aerobic fitness and obese weight status. However, the extent to which diet influences childhood cognitive function remains unclear. area of study because it encompasses cognitive processes that underlie goal-directed behavior and are orchestrated by activity within the prefrontal cortex $(3,4)$. The three interrelated, but

37 dissociable, cognitive processes thought to comprise cognitive control include inhibition (the ability to resist distractions to maintain focus), working memory (the ability to store, maintain, manipulate information to be retrieved within a brief period), and cognitive flexibility (the ability to dynamically shift attention, select information, and alter response strategy in response to changing task demands) $(5,6)$. Thus, cognitive control processes enable us to adjust our behavior

42 to changing environmental demands. Further, the prefrontal cortex exhibits a protracted developmental trajectory throughout childhood, marked by progressive and regressive changes

44 (myelination and synaptic pruning, respectively) occurring in parallel and shaped in part by the 45 child's idiosyncratic experience (3). Therefore, understanding the impact of health behaviors 46 (e.g., physical activity and diet) and their interrelated physiological markers of health (e.g., 
47 aerobic fitness and adiposity) on cognitive control process during childhood may identify 48 modifiable targets for future interventions aiming to improve cognitive functioning in children 49 (7).

Previous studies in children have associated key aspects of childhood physiological

51 health, particularly aerobic fitness, to tasks assessing inhibitory control, working memory, and

52 cognitive flexibility (8-10). These effects have been further supported by neuroimaging data

53 demonstrating that greater aerobic fitness is positively and selectively associated with neural

54 substrates subserving cognitive control (11). In addition, a relatively consistent finding is that

55 obesity is associated with cognitive deficits, especially in cognitive control, in children,

56 adolescents, and adults (12). Among prepubertal children, even following adjustment of aerobic

57 fitness, increasing adiposity or obese weight status have been shown to be negatively related to

58 children's performance on cognitive control tasks (13-16). Evidence implicating excess adiposity

59 and obese weight status in compromised cognitive control in childhood is especially concerning

60 given that approximately one-third of the children in the United States are estimated to be

61 overweight or obese. While a converging body of literature points to the beneficial effects of

62 higher aerobic fitness and maintenance of a healthy weight status for cognitive control in

63 childhood, the evidence relating specific aspects of diet intake to children's cognitive control, 64 particularly cognitive flexibility, remains limited.

Diet and cognitive function are thought to interact via multiple pathways. Diet may directly influence cognitive function by a variety of mechanisms including provision of essential

67 nutrients for brain development (17), amelioration of neuroinflammation $(18,19)$, and provision

68 of energy (20). Alternatively, impairment in cognitive control functions may contribute to poor

69 dietary choices. Indeed, cognitive control functions are thought to be necessary to regulate eating 
behaviors (21). Pertinent to the study reported herein, diets high in saturated fats (SFAs) and

71 cholesterol have been previously linked to cognitive impairment in animal studies as well as

72 human trials. Rodents consuming diets higher in saturated fats exhibit decrements on memory

73 tasks as well as neurotrophic factors important for neuronal plasticity and learning $(22,23)$.

74 Among humans, consuming a diet high in SFA during midlife is associated with an increased

75 risk for cognitive impairment in later adulthood (24). Similarly, higher dietary cholesterol intake

76 among older adults is significantly associated with an increased risk of impaired memory and

77 cognitive flexibility (25). Among children, dietary lipids have been shown to be differentially

78 associated with memory function (26) while overall diet quality was found to be positively

79 correlated with academic performance among $5^{\text {th }}$ grade students $(27)$ and children's attentional

80 inhibitory control (28). Therefore, specific dietary components may play an important role in

81 children's cognitive control. However, links between nutrients and specific cognitive domains

82 are not well characterized.

Specifically, the association of saturated fats and cholesterol intake to children's

84 cognitive flexibility has received little to no attention in the literature. Although the influence of

85 diet on inhibitory control has received considerable interest $(28,29)$, the relationship between

86 habitual diet intake and cognitive flexibility has received comparatively less attention. Tasks

87 tapping into cognitive flexibility are distinct from inhibitory control because the former requires

88 switching between two or more mental sets rather than the inhibition of a single response or the

89 suppression of interfering information $(3,30)$. Indeed, successful task switching may index a

90 higher order of information processing and action control requiring both working memory and

91 inhibitory control $(3,31)$. Previous studies have shown that aerobic fitness and obesity exert

92 contrasting effects on cognitive flexibility among prepubertal children $(10,16,32)$. Therefore, 
93 examining whether or not diet also plays a role in cognitive flexibility stands to expand our

94 understanding of modifiable lifestyle factors that may be targeted for improvement of all

95 cognitive control processes, and not only inhibitory control.

Accordingly, the study reported herein examined associations between saturated fats and

97 cholesterol intake and cognitive flexibility, assessed using a task switching paradigm, among a

98 sample of prepubertal children between 7-10-years. Our central hypothesis was that increasing

99 intake of saturated fats and dietary cholesterol would be related to poorer performance 100 indicated by lower accuracy, longer reaction times, and higher global and local switch costs - on 101 a cognitive flexibility task, following adjustment of confounding variables including 102 demographics, IQ, aerobic fitness and BMI.

104 MATERIALS AND METHODS

105 Participants

The results of this study are based on cross-sectional analysis of baseline data collected

107 from a subsample of prepubertal children between 7 and 10 years $(N=150)$ who participated in a 108 larger randomized controlled trial [Fitness Improves Thinking in Kids (FITKids; 109 NCT01334359)]. Only FITKids participants who provided diet and the switch task were 110 included in the sample studied herein. Participants' provided written assent and their legal 111 guardians' provided written informed consent in accordance with the regulations of the 112 University of Illinois Institutional Review Board. Children were screened for neurological 113 disorders, physical disabilities, psychoactive medication status, and normal or corrected-to114 normal vision. Data were also collected on 1) IQ, using the Kaufman Brief Intelligence Test (33), 
115 2) socioeconomic status (SES) as estimated based on participation in a school meal-assistance 116 program, maternal and paternal education levels, and how many parents work full-time, and 3) 117 pubertal status using the modified Tanner Staging System (34). The sample comprised of both 118 healthy weight (66\%) and overweight/obese children (34\%). Participants with low SES 119 constituted $34 \%$ of the sample and $65 \%$ were categorized as Tanner stage 1.

Diet Assessment

Dietary intake was collected using one 24-hour food recall conducted by a Registered 122 Dietitian. The recall was completed by the child with assistance from the parent. The Nutrition 123 Data Systems-Research (NDSR; Nutrition Coordinating Center, Minneapolis, MN, USA) 124 software was used to analyze dietary intake. Nutrient-level analyses were conducted using the 125 intake properties file to determine macronutrient (carbohydrate, protein, and fat) intake.

Anthropometric Assessment

Height and weight were measured using a stadiometer (Seca; model 240) and a Tanita WB-300 Plus digital scale, respectively. The mean of three measurements of height and weight were used for the analyses. The Centers for Disease Control (CDC, 2000) growth charts were used to determine each child's BMI-for-age percentile (35).

Aerobic Fitness Assessment

Cardiorespiratory fitness was assessed using a test of maximal oxygen consumption $133\left(\mathrm{VO}_{2 \max }\right)(36)$. Oxygen consumption was measured using a computerized indirect calorimetry system (ParvoMedics True Max 2400) while participants ran/walked on a motor-driven treadmill 135 at a constant speed with incremental grade increases of $2.5 \%$ grade every two minutes until the participant was no longer able to maintain the exercise intensity (36). A Polar heart rate monitor 
137 (Polar WearLink+ 31; Polar Electro, Finland) was used to measure heart rate throughout the test

138 and ratings of perceived exertion were assessed every 2 min using the children's OMNI scale

139 (37). $\mathrm{VO}_{2 \max }$ was based upon maximal effort as evidenced by (1) a peak heart rate $>185 \mathrm{bpm}$

140 (36) and a heart rate plateau (38); (2) RER > 1.0 (39); (3) a score on the children's OMNI ratings

141 of perceived exertion scale > 7 (37); and/or (4) a plateau in oxygen consumption corresponding

142 to an increase of less than $2 \mathrm{ml} / \mathrm{kg} / \mathrm{min}$ despite an increase in workload.

143

144

145

146

147

148

149

150

151

152

153

154

155

156

157 158

\section{Switch Task}

Cognitive flexibility was assessed using a color-shape task switching paradigm (40). This task requires participants to learn a set of response mappings arbitrarily assigned to a set of colors (blue and green) and shapes (circle and square), then utilize a rule-set cue (the direction of the characters arms) to flexibly shift visuospatial attention towards the correct feature set and execute the correct response mapping [see (10)]. In this task, participants completed two blocks of 60 homogeneous trials ( 1 block of color only; 1 block of shape only) in which they attended to a centrally presented character. Participants were instructed to make a left hand thumb press on a Neuroscan STIM system response pad (Compumedics, Charlotte, NC) when the character was blue (in the color condition) or a circle (in the shape condition) and a right hand thumb press when the character was green (in the color condition) or a square (in the shape condition).

During the heterogeneous condition, participants performed both the color and shape tasks together with the specific task on each trial indicated by the direction of the character's arms (arms up: respond based on the shape of the character; arms down: respond based on the color of the character). After a block of 40 practice trials, participants completed three blocks of 50 heterogeneous trials with equiprobable task occurrence and response directionality. The stimuli 
159 were $5.5 \mathrm{~cm}$ tall and $9 \mathrm{~cm}$ wide characters presented focally for $3000 \mathrm{~ms}$ on a black background 160 - or until a response was given - with a fixed inter-trial interval of $3500 \mathrm{~ms}$.

Switch task measures of interest included accuracy and reaction time with greater 162 accuracy and faster reaction times indicating superior cognitive flexibility. In addition to 163 accuracy and reaction time, global and local switch costs were calculated to index the flexible 164 modulation of cognitive control when faced with greater task demands. Global switch cost 165 analyses examined differences between homogenous and heterogeneous conditions while local 166 switch cost analyses examined differences between switch and non-switch trials during the 167 heterogeneous block condition. Global switch cost related to accuracy was defined as the 168 difference in performance between homogeneous and heterogeneous blocks (accuracy cost: 169 homogenous - heterogeneous; reaction time cost: heterogeneous - homogenous). Thus, lower 170 global switch costs reflect the greater efficiency of maintaining multiple task sets in working 171 memory as well as the selection of the task to be performed next (41). Local switch costs were

172 defined as the difference between performance on switch trials and non-switch trials within 173 heterogeneous blocks (accuracy cost: non-switch - switch; reaction time cost: switch - non174 switch). Lower local switch costs are thought to reflect greater effectiveness of cognitive control 175 processes responsible for the activation of the presently relevant task set and the deactivation of 176 the task set that was relevant on the previous trial (42). We hypothesized that intake of dietary 177 cholesterol and saturated fatty acids will be associated with greater global and local switch costs, 178 signifying increasing decrements in cognitive flexibility.

\section{Statistical Analyses}

Differences in cognitive flexibility across categorical variables (e.g., healthy vs. obese

181 weight status, SES, and sex) were conducted using an independent $t$ test. Initial Pearson's 
182 correlations assessed bivariate relationships between demographic variables and cognitive

183 flexibility measures among all participants. Subsequently, partial correlations were conducted

184 between diet variables and cognitive flexibility following adjustment of control variables (age,

185 sex, SES, K-BIT, $\mathrm{VO}_{2 \max }$, and $\left.\mathrm{BMI}\right)$. Control variables were chosen based on prior research

186 relating these factors to measures of cognitive control $(16,43)$. One-tailed $t$ tests were used

187 because this study aimed to test directional hypotheses regarding the associations between

188 cholesterol and saturated fatty acids and cognitive flexibility measures. To adjust for overall

189 energy intake, nutrient intake was normalized to intake per $1000 \mathrm{kcal}$ within participants before

190 subsequent analyses. Statistics were performed using SPSS 22 (IBM, Somers, NY).

\section{RESULTS}

Participant characteristics (demographics, weight status, and aerobic fitness) and switch

193 task performance are presented in Table 1. Mean reaction times were determined after averaging

194 correct trials. Table 2 summarizes the diet intake variables among participants. Participants

195 reported meeting approximately 52\% $(S D=9.54)$ of their energy needs from carbohydrates,

$19615.3 \%(S D=4.6)$ from proteins, 32.6\% $(S D=7.5)$ from fats, and 12.4\% $(S D=4.4)$ from saturated

197 fats.

There were no significant differences in diet intake, K-BIT, and switch task response

199 accuracy and reaction time for homogeneous and heterogeneous trials between healthy weight

200 and obese children. However, obese children exhibited greater global switch cost for accuracy

$201(P=0.02)$ compared to healthy weight children. Aerobic fitness, assessed as $\mathrm{VO}_{2 \max }$ relative to

202 body weight, was higher $(P<0.01)$ among males while females had higher BMI $(P<0.01)$. On the

203 other hand, no differences in diet intake were observed between males and females. Further,

204 there were no significant differences in K-BIT, switch task response accuracy and reaction time 
205 for homogeneous and heterogeneous trials across sex groups. However, females exhibited 206 greater global switch costs for accuracy $(P=0.03)$. Differences between high $(\mathrm{n}=61)$ and low 207 (n=51) SES groups revealed that high SES children had higher K-BIT $(P<0.01)$, $\mathrm{VO}_{2 \max }$ $208(P<0.01)$, and consumed higher protein $(P=0.03)$ while children categorized as low SES children 209 exhibited greater local switch cost for reaction time $(P=0.04)$.

Bivariate correlations were conducted to identify non-diet variables that may be associated with cognitive flexibility measures. Age was positively related to accuracy on the 212 homogeneous $(r=0.20, P<0.01)$ and heterogeneous trials $(r=0.28, P<0.01)$ and negatively related to global accuracy switch cost $(r=-0.19, P=0.01)$. Sex was related to global switch cost for 214 accuracy $(r=0.18, P=0.01)$ such that males had a lower global switch cost. K-BIT was positively 215 related to heterogeneous accuracy $(r=0.23, P<0.01)$ and negatively related to global switch cost 216 for accuracy $(r=-0.19, P=0.01)$, signifying that greater IQ was associated with the ability to 217 maintain accuracy when task demands increased. BMI was related to greater global switch cost 218 for accuracy $(r=0.20, P=0.01)$ whereas $\mathrm{VO}_{2 \max }$ was related to greater accuracy on heterogeneous 219 trials $(r=0.25, P<0.01)$ and negatively related to global switch cost for accuracy $(r=-0.26$, $220 \quad P<0.01)$.

221 Results of the partial correlations between the diet variables and switch task performance 222 - following adjustment of age, sex, K-BIT, SES, $\mathrm{VO}_{2 \max }$, and BMI - are presented in Table 3. 223 Following adjustment of covariates, total fats $(r=0.20, P<0.01)$, saturated fats $(r=0.23, P<0.01)$, 224 and cholesterol $(r=0.17, P=0.02)$ were related to greater local switch cost for accuracy. Saturated 225 fats were related to increased heterogeneous reaction time $(r=0.17, P=0.02)$ and global switch 226 cost for reaction time $(r=0.14, P=0.04)$. Cholesterol intake was related to increased local switch 227 for both accuracy $(r=0.17, P=0.02)$ and reaction time $(r=0.14, P=0.04)$. 


\section{DISCUSSION}

Previous studies have shown that cognitive flexibility is positively associated with aerobic fitness and negatively related to adiposity among prepubertal children $(10,16)$. However,

232 data relating dietary components to childhood cognitive flexibility is limited. The current study

233 provides novel evidence linking diet intake to cognitive flexibility among prepubertal children.

234 The major findings were that fats, specifically saturated fats, were related to longer reaction time 235 during the heterogeneous trials - the trial type requiring greater cognitive flexibility - and greater 236 global switch cost for reaction time. Thus, increasing intake of saturated fats was associated with 237 impaired ability to maintain multiple task sets in working memory as well as the selection of the 238 subsequent task (44). Further, increasing intake of dietary cholesterol was related to greater local 239 switch costs for both accuracy and reaction time, reflecting lower efficiency of executive control 240 processes responsible for the activation of the presently relevant task set and the deactivation of 241 the task set that was relevant on the previous trial (45). Collectively, these data are among the 242 first to indicate that children consuming diets higher in saturated fats and cholesterol exhibit 243 compromised ability to flexibly modulate their cognitive flexibility, particularly when faced with 244 greater task demands.

A broader implication of this study was that it provided evidence identifying cognitive flexibility as another core cognitive control process that may be susceptible to the detrimental

247 effects of saturated fats and cholesterol intake. Cognitive flexibility is important because it 248 comprises the ability to switch perspectives in daily life (e.g. viewing a problem from the point 249 of view of others or from a different direction) and involves being flexible enough to adjust to 250 changing demands or priorities. This capacity to switch perspectives depends on the child's 
251 ability to inhibit (or deactivate) a previous perspective and load into working memory (or 252 activate) a different perspective (31), integrating both inhibitory and working memory abilities. 253 However, relative to other cognitive control processes, cognitive flexibility develops later, with 254 the ability to flexibly switch, on a trial-by-trial basis, not emerging until 7 to 9 years of age (46, 255 47). This later emergence may partially explain the limited number of studies investigating the 256 behavioral correlates of cognitive flexibility in childhood.

Nevertheless, emerging evidence demonstrates that the provision of physical activity can 258 improve cognitive flexibility in both children and adults $(10,48)$. Further, increasing BMI and 259 excess fat mass has been related to poorer performance on cognitive flexibility tasks among 260 prepubertal children $(16,32)$. In the current study, BMI was also related to cognitive flexibility, 261 such that children with higher BMI exhibited poorer ability to upregulate their cognitive control 262 when faced with greater task demands.

Although data relating diet to cognitive flexibility is limited, previous studies have 264 correlated specific nutrients to inhibitory control and memory function in children. Baym et al. 265 (2014) observed that increasing intake of omega-3 fats was selectively and positively related to 266 hippocampal-dependent relational memory among prepubertal children (26). In contrast, 267 saturated fats were negatively related to both hippocampal-dependent and hippocampal268 independent forms of memory, suggesting a generalized negative effect of saturated fats on 269 memory function (26). Among a similar sample of children, Khan et al. (2015) observed that 270 overall diet quality and dietary fiber were positively related to inhibitory control while total fat 271 intake was negatively related to the ability to suppress interference when cognitive demands 272 were increased (28). Further, increasing intake of dietary cholesterol has been shown to be 273 negatively related to children's performance on a digit span task (49). Similar to Baym et al. 
274 (2014), the current study's findings support a generalized negative association between saturated 275 fats and cognitive function since increasing saturated fats were related to both global and local 276 switch costs. On the other hand, increasing dietary cholesterol was only related to greater local 277 switch costs, reflecting selective association with lower efficiency of cognitive control processes. 278 Therefore, the findings from the current study complement previous work by detecting that 279 cognitive flexibility is another core cognitive control process that may be susceptible to the 280 deleterious effects of saturated fats while identifying dietary cholesterol as a negative correlate of 281 cognitive flexibility in children as well.

Given the association between saturated fat and cholesterol intake and metabolic risk, it is 283 curious that the influence of these nutrients on childhood cognitive flexibility has not been 284 directly investigated. Intake of saturated fats has been linked - primarily through increased 285 concentration of low-density lipoprotein (LDL) - to an increased risk for cardiovascular disease 286 (50). However, the deleterious effects of saturated fats on cognitive function have been observed 287 in rodent studies as well as human trials. Rats fed $20 \%$ of their calories from saturated fats 288 exhibit decrements in learning and cognitive function, relative to rats consuming 289 monounsaturated, or polyunsaturated fats (51). Subsequent studies indicated that the mechanism 290 underling this effect may involve saturated fat-induced oxidative stress and reduced BDNF 291 resulting in compromised synaptic plasticity (52). Further, saturated fats increase insulin 292 resistance in the brain (53) and diminish the integrity of the blood brain barrier (54). When fed in 293 combination with cholesterol, saturated fat diets of up to $10 \%$ have been shown to reduce 294 dendritic integrity in rats (55). Among adult humans, higher dietary cholesterol intake was 295 significantly associated with an increased risk of impaired memory and cognitive flexibility. 296 However, the effects of saturated fat intake on impaired memory, speed, and cognitive flexibility 
297 was not statistically significant (56). Further, use of drugs that lower blood cholesterol 298 concentrations (e.g. statins) may be associated with a lower risk of dementia among older adults 299 (57). However, among children, the evidence remains mixed, with some studies finding 300 associations between saturated fats and cholesterol and cognitive functioning and others not 301 observing a relationship $(26,28,49)$. Nevertheless, consistent with data from animal studies and 302 adult trials, findings from this study indicate that increasing saturated fat intake and dietary 303 cholesterol may exert independent effects on cognitive functioning in children as well.

Although the current study provides support for the negative association between saturated fats and dietary cholesterol and cognitive flexibility, there are several limitations worth 306 considering. Given that these results are based on cross-sectional analyses, we cannot make 307 statements of causality. While it is assumed that variability in cognitive flexibility was driven by 308 the diet measures, it is possible that the opposite may be true, such that lower cognitive 309 flexibility may be implicated in poorer dietary choices. Future longitudinal and/or intervention 310 studies examining the effects of saturated fat and cholesterol intake on cognitive flexibility could 311 provide definitive support for the directionality of the associations observed in the current study. 312 Further, although there is validity in using one 24-hour recall to assess diet intake among groups 313 of people, additional studies that utilize other multiple diet assessment techniques such as food 314 records or food frequency questionnaires would provide further support for this work. In 315 addition, our analyses did not account for all nutrients consumed. Therefore, we cannot exclude 316 the possibility that micronutrients (i.e., vitamins and minerals) may have contributed to our 317 results. Finally, future studies should assess serum or plasma markers of metabolism and 318 inflammation to uncover the mechanisms that may underlie these observations. Nevertheless, the 319 strengths of the current study included the adjustment of several key covariates known to 
320 influence childhood cognitive function e.g., age, sex, SES, IQ, aerobic fitness, and BMI.

321 Therefore, the finding that specific dietary components significantly correlated with cognitive

322 flexibility - following adjustment of key covariates - highlights the importance of assessing

323 saturated fat and cholesterol intake along with cognitive flexibility outcomes in future studies

324 among child populations.

\section{CONCLUSIONS}

Given that rapid rise and persistence of the childhood obesity epidemic, the detrimental

328 effects of physical inactivity and excess fat mass on childhood cognitive function are becoming

329 increasingly recognized. However, the extent to which specific dietary components influence 330 cognitive functioning among children, without clinical nutrient deficiencies, remains unclear.

331 The current study provides novel evidence relating saturated fats and dietary cholesterol to

332 cognitive flexibility among prepubertal children. Critically, we demonstrate that increasing 333 intake of saturated fats and cholesterol is associated with the compromised ability to flexibly 334 modulate cognitive control, particularly when task demands are increased. Diet 335 recommendations for lowering saturated fat intake and dietary cholesterol have been shown to 336 result in improved cardiovascular health among children and adults. The cross-sectional findings 337 from this study provide preliminary support for future intervention studies that may aim to 338 optimize cognitive health by reducing saturated fat and cholesterol intake among pediatric 339 populations. 


\section{Acknowledgements}

343 This study was supported by NIH grants (HD055352) and USDA NIFA grant (2011-67001-

344 30101). We thank the participants, their families, and the Urbana School District 116 for

345 participating in the study. We thank Bonnie Hemrick for her assistance in participant recruitment

346 and randomization. We would also like to thank Bridget Schuld, Ashley Ejnik, and Alexis Tiller

347 for assisting with the analysis of the diet recall data. 


\section{References}

1. Lenroot RK, Giedd JN. Brain development in children and adolescents: insights from anatomical magnetic resonance imaging. Neuroscience \& Biobehavioral Reviews. 2006;30(6):718-29.

2. Luna B. Developmental changes in cognitive control through adolescence. Adv Child Dev Behav. 2009;37:233-78.

3. Best JR, Miller PH. A developmental perspective on executive function. Child Dev. 2010;81(6):1641-60.

4. Shimamura AP. The role of the prefrontal cortex in dynamic filtering. Psychobiology. 2000;28(2):207-18.

5. Davidson MC, Amso D, Anderson LC, Diamond A. Development of cognitive control and executive functions from 4 to 13 years: Evidence from manipulations of memory, inhibition, and task switching. Neuropsychologia. 2006;44(11):2037-78.

6. Kane MJ, Engle RW. The role of prefrontal cortex in working-memory capacity, executive attention, and general fluid intelligence: An individual-differences perspective. Psychon Bull Rev. 2002;9(4):637-71.

7. Miller EK, Cohen JD. An integrative theory of prefrontal cortex function. Annu Rev Neurosci. 2001;24(1):167-202.

8. Kamijo K, Pontifex MB, O’Leary KC, Scudder MR, Wu C, Castelli DM, et al. The effects of an afterschool physical activity program on working memory in preadolescent children.

Developmental science. 2011;14(5):1046-58.

9. Pontifex MB, Raine LB, Johnson CR, Chaddock L, Voss MW, Cohen NJ, et al. Cardiorespiratory fitness and the flexible modulation of cognitive control in preadolescent children. J Cogn Neurosci. 2011;23(6):1332-45.

10. Hillman CH, Pontifex MB, Castelli DM, Khan NA, Raine LB, Scudder MR, et al. Effects of the FITKids randomized controlled trial on executive control and brain function. Pediatrics. 2014 Oct;134(4):e1063-71.

11. Chaddock L, Erickson KI, Prakash RS, VanPatter M, Voss MW, Pontifex MB, et al. Basal ganglia volume is associated with aerobic fitness in preadolescent children. Dev Neurosci. 2010;32(3):249-56.

12. Smith E, Hay P, Campbell L, Trollor J. A review of the association between obesity and cognitive function across the lifespan: implications for novel approaches to prevention and treatment. Obesity Reviews. 2011;12(9):740-55. 
13. Kamijo K, Pontifex MB, Khan NA, Raine LB, Scudder MR, Drollette ES, et al. The Negative Association of Childhood Obesity to Cognitive Control of Action Monitoring. Cerebral Cortex. 2012.

14. Kamijo K, Pontifex MB, Khan NA, Raine LB, Scudder MR, Drollette ES, et al. The association of childhood obesity to neuroelectric indices of inhibition. Psychophysiology. 2012;49(10):1361-71.

15. Kamijo K, Pontifex MB, Khan NA, Raine LB, Scudder MR, Drollette ES, et al. The negative association of childhood obesity to cognitive control of action monitoring. Cereb Cortex. 2014 Mar;24(3):654-62.

16. Pontifex MB, Kamijo K, Scudder MR, Raine LB, Khan NA, Hemrick B, et al. The differential association of adiposity and fitness with cognitive control in preadolescent children. Monogr Soc Res Child Dev. 2014;79(4):72-92.

17. Georgieff MK. Nutrition and the developing brain: nutrient priorities and measurement. Am J Clin Nutr. 2007;85(2):614S-20S.

18. Sherry CL, Kim SS, Dilger RN, Bauer LL, Moon ML, Tapping RI, et al. Sickness behavior induced by endotoxin can be mitigated by the dietary soluble fiber, pectin, through up-regulation of IL-4 and Th2 polarization. Brain Behav Immun. 2010;24(4):631-40.

19. Jang S, Dilger RN, Johnson RW. Luteolin inhibits microglia and alters hippocampaldependent spatial working memory in aged mice. J Nutr. 2010 Oct;140(10):1892-8.

20. Hoyland A, Lawton CL, Dye L. Acute effects of macronutrient manipulations on cognitive test performance in healthy young adults: a systematic research review. Neurosci Biobehav Rev. 2008;32(1):72-85.

21. Guerrieri R, Nederkoorn C, Stankiewicz K, Alberts H, Geschwind N, Martijn C, et al. The influence of trait and induced state impulsivity on food intake in normal-weight healthy women. Appetite. 2007;49(1):66-73.

22. Molteni R, Barnard R, Ying Z, Roberts C, Gomez-Pinilla F. A high-fat, refined sugar diet reduces hippocampal brain-derived neurotrophic factor, neuronal plasticity, and learning. Neuroscience. 2002;112(4):803-14.

23. Kanoski SE, Zhang Y, Zheng W, Davidson TL. The effects of a high-energy diet on hippocampal function and blood-brain barrier integrity in the rat. J Alzheimer's Dis. 2010;21(1):207-19.

24. Eskelinen MH, Ngandu T, Helkala E, Tuomilehto J, Nissinen A, Soininen H, et al. Fat intake at midlife and cognitive impairment later in life: a population-based CAIDE study. Int J Geriatr Psychiatry. 2008;23(7):741-7. 
25. Kalmijn S, Van Boxtel M, Ocke M, Verschuren W, Kromhout D, Launer L. Dietary intake of fatty acids and fish in relation to cognitive performance at middle age. Neurology.

2004;62(2):275-80.

26. Baym CL, Khan NA, Monti JM, Raine LB, Drollette ES, Moore RD, et al. Dietary lipids are differentially associated with hippocampal-dependent relational memory in prepubescent children. Am J Clin Nutr. 2014;99(5):1-8.

27. Florence MD, Asbridge M, Veugelers PJ. Diet Quality and Academic Performance*. J Sch Health. 2008;78(4):209-15.

28. Khan NA, Raine LB, Drollette ES, Scudder MR, Kramer AF, Hillman CH. Dietary Fiber Is Positively Associated with Cognitive Control among Prepubertal Children. J Nutr. 2015 Jan;145(1):143-9.

29. Jasinska AJ, Yasuda M, Burant CF, Gregor N, Khatri S, Sweet M, et al. Impulsivity and inhibitory control deficits are associated with unhealthy eating in young adults. Appetite. 2012;59(3):738-47.

30. Crone EA, Somsen RJ, Zanolie K, Van der Molen, Maurits W. A heart rate analysis of developmental change in feedback processing and rule shifting from childhood to early adulthood. J Exp Child Psychol. 2006;95(2):99-116.

31. Diamond A. Executive functions. Annu Rev Psychol. 2013;64:135-68.

32. Wirt T, Schreiber A, Kesztyüs D, Steinacker JM. Early Life Cognitive Abilities and Body Weight: Cross-Sectional Study of the Association of Inhibitory Control, Cognitive Flexibility, and Sustained Attention with BMI Percentiles in Primary School Children. Journal of Obesity. 2014.

33. Kaufman AS. K-BIT: Kaufman brief intelligence test. American Guidance Service Circle Pines, MN; 1990.

34. Tanner JM. Growth at adolescence. Oxford: Blackwell Scientific Publications; 1962.

35. Kuczmarski RJ, Ogden CL, Grummer-Strawn LM, Flegal KM, Guo SS, Wei R, et al. CDC growth charts: United States. Adv Data. 2000(314):1-27.

36. Armstrong L. ACSM's guidelines for exercise testing and prescription/American College of Sports Medicine. 7th ed. Philadelphia: Lippincott Williams \& Wilkins, Philadelphia; 2006.

37. Utter AC, Roberson RJ, Nieman DC, Kang J. Children's OMNI Scale of Perceived Exertion: walking/running evaluation. Med Sci Sports Exerc. 2002;34(1):139-44.

38. Freedson PS, Goodman TL, editors. Measurement of oxygen consumption. Champaign, IL: Human Kinetics; 1993. 
39. Bar-Or O. Pediatric sports medicine for the practitioner: From physiologic principles to clinical applications. Springer-Verlag New York:; 1983.

40. Espy KA. The Shape School: Assessing executive function in preschool children. Dev Neuropsychol. 1997;13(4):495-9.

41. Kray J, Lindenberger U. Adult age differences in task switching. Psychol Aging. 2000;15(1):126.

42. Meiran N. Reconfiguration of processing mode prior to task performance. Journal of Experimental Psychology: Learning, Memory, and Cognition. 1996;22(6):1423.

43. Kamijo K, Khan NA, Pontifex MB, Scudder MR, Drollette ES, Raine LB, et al. The relation of adiposity to cognitive control and scholastic achievement in preadolescent children. Obesity. 2012;20(12):2406-11.

44. Kray J, Lindenberger U. Adult age differences in task switching. Psychol Aging. 2000;15(1):126.

45. Meiran N. Reconfiguration of processing mode prior to task performance. Journal of Experimental Psychology: Learning, Memory, and Cognition. 1996;22(6):1423.

46. Davidson MC, Amso D, Anderson LC, Diamond A. Development of cognitive control and executive functions from 4 to 13 years: Evidence from manipulations of memory, inhibition, and task switching. Neuropsychologia. 2006;44(11):2037-78.

47. Gupta R, Kar BR, Srinivasan N. Development of task switching and post-error-slowing in children. Behavioral and Brain Functions. 2009;5(38):1-13.

48. Masley S, Roetzheim R, Gualtieri T. Aerobic exercise enhances cognitive flexibility. Journal of clinical psychology in medical settings. 2009;16(2):186-93.

49. Zhang J, Hebert JR, Muldoon MF. Dietary Fat Intake Is Associated with Psychosocial and Cognitive Functioning of School-Aged Children in the United States. J Nutr. 2005 August 01;135(8):1967-73.

50. Siri-Tarino PW, Sun Q, Hu FB, Krauss RM. Saturated fat, carbohydrate, and cardiovascular disease. Am J Clin Nutr. 2010 Mar;91(3):502-9.

51. Greenwood CE, Winocur G. Cognitive impairment in rats fed high-fat diets: a specific effect of saturated fatty-acid intake. Behav Neurosci. 1996;110(3):451.

52. Wu A, Ying Z, Gomez-Pinilla F. The interplay between oxidative stress and brain-derived neurotrophic factor modulates the outcome of a saturated fat diet on synaptic plasticity and cognition. Eur J Neurosci. 2004;19(7):1699-707. 
53. Alsaif MA, Duwaihy M. Influence of dietary fat quantity and composition on glucose tolerance and insulin sensitivity in rats. Nutr Res. 2004;24(6):417-25.

54. Kanoski SE, Zhang Y, Zheng W, Davidson TL. The effects of a high-energy diet on hippocampal function and blood-brain barrier integrity in the rat. J Alzheimer's Dis. 2010;21(1):207-19.

55. Granholm A, Bimonte-Nelson HA, Moore AB, Nelson ME, Freeman LR, Sambamurti K. Effects of a saturated fat and high cholesterol diet on memory and hippocampal morphology in the middle-aged rat. J Alzheimer's Dis. 2008;14(2):133-45.

56. Kalmijn S, van Boxtel MP, Ocke M, Verschuren WM, Kromhout D, Launer LJ. Dietary intake of fatty acids and fish in relation to cognitive performance at middle age. Neurology. 2004 Jan 27;62(2):275-80.

57. Jick H, Zornberg GL, Jick SS, Seshadri S, Drachman DA. Statins and the risk of dementia. The Lancet. 2000;356(9242):1627-31.

58. Hellwig JP, Otten JJ, Meyers LD. Dietary Reference Intakes:: The Essential Guide to Nutrient Requirements. National Academies Press; 2006.

59. Institute of Medicine (US). Panel on Macronutrients. Dietary reference intakes for energy, carbohydrate, fiber, fat, fatty acids, cholesterol, protein, and amino acids. ; 2005. 
Table 1. Participant characteristics and switch task performance

\begin{tabular}{|c|c|c|}
\hline & Mean & SD \\
\hline Age, years & $8.8(0.6)$ & 0.6 \\
\hline Socioeconomic Status, \% & & \\
\hline Low & 34.0 & \\
\hline Medium & 25.0 & \\
\hline High & 41.0 & \\
\hline Pubertal Timing, $\%$ & & \\
\hline Tanner Stage 1 & 65.0 & \\
\hline Tanner Stage 2 & 35.0 & \\
\hline BMI, $k g / m^{2}$ & 18.5 & 3.9 \\
\hline BMI-for-age, \%tile & 65.4 & 28.3 \\
\hline$<85^{\text {th }}$ & 66.0 & \\
\hline$\geq 85^{\text {th }}$ & 34.0 & \\
\hline $\mathrm{VO}_{2 \max }, \mathrm{kg} / \mathrm{ml} / \mathrm{min}$ & 39.0 & 6.8 \\
\hline Intelligence Quotient & 112.0 & 14.2 \\
\hline Switch Task & & \\
\hline Response Accuracy, \% & & \\
\hline Homogenous Trials & 86.7 & 8.1 \\
\hline Heterogeneous Trials & 66.3 & 15.0 \\
\hline Global Switch Costs & 20.4 & 13.6 \\
\hline Local Switch Costs & 2.0 & 7.4 \\
\hline Reaction Time, $m s$ & & \\
\hline Homogenous Trials & 807.1 & 160.8 \\
\hline Heterogeneous Trials & 1439.2 & 252.8 \\
\hline Global Switch Costs & 632.1 & 250.2 \\
\hline Local Switch Costs & 174.3 & 164.4 \\
\hline
\end{tabular}


Table 2. Dietary intake among study participants

\begin{tabular}{l|c|c}
\hline & Mean (SD) & RDA/AI \\
\hline Energy, $k c a l$ & $1982.6(920.0)$ & $1593-2043^{2}$ \\
Carbohydrate, $g$ & $262.3(126.6)$ & 130 \\
Total Fats, $g$ & $73.9(39.6)$ & 31 \\
Protein, $g$ & $73.0(35.7)$ & 34 \\
Saturated Fats, $g$ & $28.1(17.1)$ & ND \\
Cholesterol, $m g$ & $218.3(148.2)$ & ND \\
\hline
\end{tabular}

${ }^{\mathrm{l}}$ Recommended values based on the Dietary Reference Intakes for Energy, Carbohydrate. Fiber, Fat, Fatty Acids, Cholesterol, Protein, and Amino Acids (2002/2005)

${ }^{2}$ Based on energy intake recommendations for sedentary - active children $(58,59)$

RDA, recommended dietary allowance; AI, adequate intake; ND, not determinable 
Table 3. Partial correlations between diet variables and cognitive flexibility measures

\begin{tabular}{c|r|c|c|c}
\hline & & Total Fats & Saturated Fats & Cholesterol \\
\hline Response Accuracy & & & & \\
Homogenous Trials & $\mathrm{r}$ & 0.01 & 0.03 & 0.12 \\
& $P$ & 0.47 & 0.36 & 0.07 \\
Heterogeneous Trials & $\mathrm{r}$ & 0.03 & -0.04 & 0.06 \\
& $P$ & 0.37 & 0.32 & 0.22 \\
Global Switch Costs & $\mathrm{r}$ & -0.03 & 0.06 & 0.01 \\
& $P$ & 0.38 & 0.24 & 0.47 \\
Local Switch Costs & $\mathrm{r}$ & $0.20^{* *}$ & $0.23^{* *}$ & $0.17^{*}$ \\
& $P$ & $<0.01$ & $<0.01$ & 0.02 \\
Reaction Time & & & & 0.03 \\
Homogenous Trials & $\mathrm{r}$ & 0.04 & 0.06 & 0.35 \\
Heterogeneous Trials & $P$ & 0.31 & 0.23 & 0.07 \\
Global Switch Costs & $P$ & 0.12 & $0.17^{*}$ & 0.17 \\
Local Switch Costs & $\mathrm{r}$ & 0.09 & 0.02 & 0.06 \\
& $P$ & 0.14 & $0.14^{*}$ & 0.24 \\
& $\mathrm{r}$ & -0.01 & 0.04 & $0.14^{*}$ \\
\hline
\end{tabular}

Control variables included age, sex, SES, K-BIT, $\mathrm{VO}_{2 \max }$, and BMI

**Significance $P<0.01$ (one tailed)

$*$ Significance $P<0.05$ (one tailed) 